\title{
Alignment Influence on 3D Anthropometric Data Clustering
}

\author{
Jianwei Niu ${ }^{1}$, Zhizhong $\mathrm{Li}^{*}{ }^{1}$ and Gavriel Salvendy ${ }^{1,2}$ \\ ${ }^{1}$ Department of Industrial Engineering, Tsinghua University, Beijing, 100084, China \\ ${ }^{2}$ School of Industrial Engineering, Purdue University, West Lafayette, IN 47907, USA
}

\begin{abstract}
Shape analysis and comparison is important to sizing system for the design of many products which require close fitting contact with the human body. However, the choice of the alignment reference can profoundly influence the shape analytical results. The objective of this case study is to demonstrate that the statistical results of the threedimensional (3D) upper heads could be different if different alignment approaches are used for the data analysis. Taking a data set of 432 upper heads as an example, this paper addressed the influence on data analysis of two alignment approaches, i.e., aligned at the centroid and aligned at the head top. K-means clustering was applied on block-based distance vectors of the upper head samples to classify the population into categories based on their shapes. Cluster membership variation of different alignment methods was examined. Results indicated that the reference can greatly influence k-means clustering results of 3D anthropometric data. Thus, alignment reference should be carefully chosen according to the specific requirements of an application.
\end{abstract}

Keywords: Three dimensional anthropometry, clustering, sizing, alignment.

\section{INTRODUCTION}

For wearing products which need close fitting, they must be designed to well match the human body surfaces of the users [1-5]. Sizing method is regarded as one of the most important factor influencing the fit and performance of products [6]. 3D anthropometry surveys, such as the CAESAR project [7], can provide tremendous amount of shape information. Researchers have utilized 3D anthropometric data in the design of helmet [8], human-harness [9] and hand tools [10], etc. However, few studies have been reported regarding the effects of the selection of alignment methods on 3D anthropometric shape comparison.

The selection of the alignment reference can profoundly influence analytical results [11]. 3D forms should be properly positioned and oriented according to a predefined alignment reference for shape comparison. During the scanning, the subjects were asked to keep the same orientation and posture. In spite of this, 3D scans were usually not aligned properly. Cai et al. [12] argued different alignment reference for translation alignment could result in different object shapes if 3D anthropometric data are processed for percentile values based on coordinates. To avoid unintended errors in generating coordinates-based statistics, extra caution should be paid in selecting the alignment references. In our view, the reference must be coincident with the design requirements of a specific application.

In our previous work, a 3D anthropometric data shape clustering method has been developed [13]. In our method, $\mathrm{K}$-means clustering was performed on the proposed block

*Address correspondence to this author at the Department of Industrial Engineering, Tsinghua University, Beijing, 100084, China; Tel: +86-1062773923; Fax: +86-10-62794399; E-mail: zzli@tsinghua.edu.cn distance based vectors of 3D samples to segment a population into groups according to size and shape simultaneously. In this paper, we present a case study to examine the influence of alignment using this 3D shape clustering method.

\section{METHODOLOGY}

The first step of this study was the obtaining and preprocessing of the data samples. Then alignment methods were determined for the later clustering comparison. Before clustering, the raw data should be converted to shape descriptors. Finally, a criterion, Membership Accuracy Rate (CMAR), was proposed to quantify the clustering difference between the two alignment methods. This section presents all the above steps.

\subsection{D head samples}

In this study, the 3D head data collection was completed by a Chinese military institute in 2002 . The head cross section scans of young male Chinese soldiers (aged from 19 to 23) were collected by a medical CT scan with a low dosage [14]. The scanning was performed from the top of the head to the neck. The number of CT slices of each head sample ranges from 45 to 60 , and the slice distance is $5 \mathrm{~mm}$. The raw data were row points of the outer contour in each slice. The data of 432 heads were examined in this study. The point number of one head sample is about 50,000. Under the CAD software Unigraphics, noise data on each cross section were removed manually by visual check by the same investigator. Here noise data refer to those points more than $2 \mathrm{~mm}$ away from the section curve.

For helmet wearers, the optimum location for protection of the head is to have the opening of the helmet for viewing just above the eyebrows [15]. The upper head was assumed as the surface area of the whole head above the glabella, which is positioned just above and between the eyebrows. 
Specifically, for our samples, data for each upper head were extracted from the upper $100 \mathrm{~mm}$ height of a whole head. This selection criterion was based on the average distance from the top of the head to the glabella.

\subsection{Alignment}

For upper head shape analysis, the following two alignment methods were compared in this study, i.e., aligned at the head top and aligned at the centroid of the upper head.

For alignment based on centroid, the centers of the mass of all sample heads were first calculated. Then all the heads were aligned by translating their centers of the mass to the origin in the Cartesian coordinate system. For each head sample, adjust the orientation of the head so that the $\mathrm{Z}$ axes approximately points from the center to the top of the head, while the $\mathrm{Y}$ axes from the center to the nose. Next, a vector in $\mathrm{X}-\mathrm{Y}$ plane starting from the center of the mass and pointing to the nose-tip was defined. All heads were rotated by $\mathrm{Z}$ axes to make the defined vectors aligned with the positive direction of the $\mathrm{Y}$ axes. Finally, by visual check on all the samples, one sample was chosen as the appropriate reference for global registration of all other samples. Global registration technique of 3D surface was adopted to calculate the orientation angles by $\mathrm{X}$ and $\mathrm{Y}$ axis to make all head samples having similar orientation. This was done with the aid of the Rapidform $^{\mathrm{TM}}$ software (www.rapidform.com).

For alignment based on head top, following the alignment based on centroid, all samples were translated along $\mathrm{Z}$ axes in order to make the head tops of all samples to have the same $\mathrm{Z}$ coordinate.

After alignment, each slice of each head sample was normalized to have 180 points by equal azimuth angle sampling.

\subsection{Block Distance-Based Vector Descriptor for Sample Surfaces}

The shape information of each sample is written in the form of a compact descriptor based on our proposed block division technique. The size of the descriptor is independent of the original sample size. Such block distance-based vector descriptor was used as the input of later k-means clustering.

We used the inscribed surface of all the samples as the comparison reference surface. The inscribed surface and all sample surfaces were divided into a predefined number of blocks, namely $m$. The distance between a sample surface $i$ and the inscribed surface on $k$ th block, namely $S_{i}(k)$, can be constructed with two parts, namely $S_{i, 1}(k)$ and $S_{i, 2}(k)$, as

$S_{i, 1}(k)=\sum_{j=1}^{n_{k}} \operatorname{dis}\left(p_{k, j}\right), k=1,2,3, \ldots, m$

where $p_{k, j}$ is the $j$ th point, and $n_{k}$ represents the number of points falling into the $k$ th block. $S_{i, 2}(k)$ can be calculated as

$S_{i, 2}(k)=\sum_{j=1}^{n_{k}}\left|\operatorname{dis}\left(p_{k, j}\right)-\frac{S_{i, 1}(k)}{n_{k}}\right|, k=1,2,3, \ldots, m$

$S_{2}$ describes the geometric variation in the corresponding local areas. In both calculations of $S_{i, 1}(k)$ and $S_{i, 2}(k)$, dis $\left(p_{k, j}\right)$ denotes the Euclidean distance between two corresponding points.

From the definitions it can be seen that $S_{i, 1}(k)$ mainly reflect size difference of two paired blocks, while $S_{i, 2}(k)$ mainly reflect the local shape difference. In such a way, the shape of a surface can be characterized by a vector $\left(S_{i, 1}(1)\right.$, $\left.S_{i, 2}(1), S_{i, 1}(2), S_{i, 2}(2), \ldots, S_{i, 1}(m), S_{i, 2}(m)\right)$. In the case study, each head sample was divided into 20 blocks.

\subsection{K-Means Clustering}

K-means clustering was adopted in this study to classify a population into several groups. For k-means clustering, the number of the clusters should be specified beforehand. The latest Chinese national standard of 3D craniofacial dimensions for male soldiers partitioned the craniofacial data sets into seven standard head models. In order for the ease of comparison between the shape analysis results of our proposed method and the method provided by the national military standard, the number of $\mathrm{K}$ for the clustering on upper head data was set as seven in the case study.

\subsection{Cluster Membership Accuracy Rate}

K-means clustering based on block distance vectors was applied to the two data sets corresponding to the two alignment references. Cluster Membership Accuracy Rate (CMAR) was adopted to indicate the difference of clustering results under the two alignment methods. It is defined as follows,

$C M A R_{c}=\frac{N_{c}}{N}$

where $N_{c}$ is the number of samples under centroid alignment consistently grouped when compared with the clustering results under head top alignment, and $N$ is the total sample size.

\section{RESULTS}

\subsection{Block Division of Samples}

In the last section, we proposed a novel block distancebased vector descriptor to represent the 3D shape. Block division is the first step to obtain such a block distance-based vector descriptor for each sample. Under the CAD software Unigraphics, the block division of an upper head sample is shown in Fig. (1). Based on the definition of NURBS, each surface of the upper head samples was divided into a predefined number of blocks. As shown in the following figure, the data points lying on the upper head surface were grouped into $4 \times 5$ ( 4 in the vertical direction and 5 in the horizontal direction) patches, i.e. so-called "blocks". After the block division, by using our definition of distance between corresponding blocks, each upper head will be converted into a novel block distance-based vector descriptor.

\subsection{Sizing Results}

The clustering results and CMAR under the two alignment methods are summarized in Table 1 . The cluster sizes under head top alignment are 34, 45, 55, 73, 74, 89 and 62 respectively. The corresponding cluster sizes (the number of 


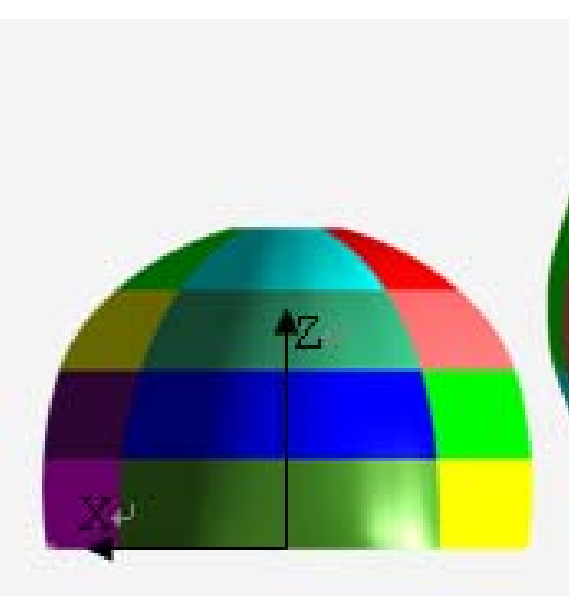

(a) front view

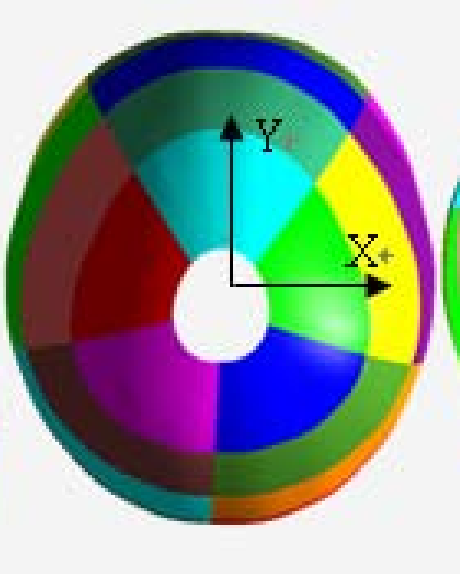

(b) top view

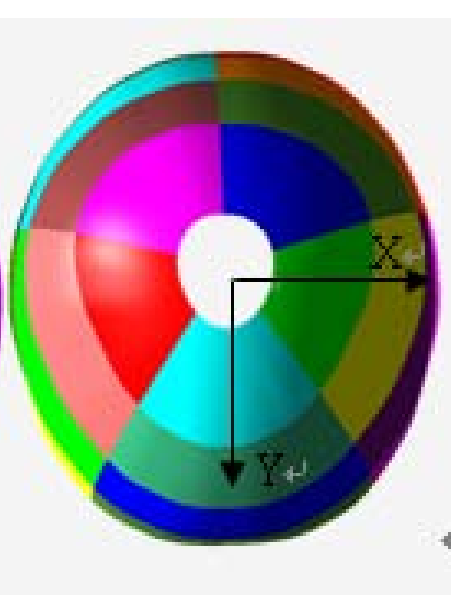

(c) bottom view

Fig. (1). Block division of a sample.

samples belong to a cluster) under centroid alignment are 37 , $53,79,62,73,75$ and 53 respectively. The number of improper clustering samples is 229 (total sample size is 432). The CMAR value is $229 / 432 * 100 \%=53 \%$.

Table 1. Cluster Membership Statistics of Upper Heads $(N=\mathbf{4 3 2})$

\begin{tabular}{|c|c|c|}
\hline \multirow{2}{*}{ Cluster ID } & \multicolumn{2}{|c|}{ Cluster Size } \\
\cline { 2 - 3 } & Aligned at Head Top & Aligned at Centroid \\
\hline \hline 1 & 34 & 37 \\
\hline 2 & 45 & 53 \\
\hline 3 & 55 & 79 \\
\hline 4 & 73 & 62 \\
\hline 5 & 74 & 73 \\
\hline 6 & 89 & 75 \\
\hline 7 & 62 & 53 \\
\hline \multicolumn{2}{|c|}{229} \\
\hline Samples & 53.0 \\
\hline \multicolumn{2}{|c|}{ CMAR (\%) } \\
Note: CMAR - Cluster Membership Accuracy Rate. \\
\hline
\end{tabular}

\section{DISCUSSIONS}

The purpose of this study was to address the influence of alignment approaches on the statistic results of 3D anthropometric data.

Various alignment methods have been used in the anthropometric studies. For helmet accommodation, the heads must be oriented into an alignment system so that they all conform to a coincident axis system [15]. This can be done by aligning two or three anatomical landmarks as described by Huggins [16] or by aligning the head within a helmet system as described by Robinette and Whitestone [17]. For a long time, Frankfurt plane has been used as alignment reference. The head is said to be in the Frankfort plane when the right and left tragion as well as right infraorbitales are aligned in a plane perpendicular to the rear headboard and parallel to the top headboard [15].
Another kind of approach is to define a vector chosen as the reference for each head. Bradtmiller and Beecher [18] defined a midpoint for vector development on a plane which lies just above the ears and the glabella. On this plane, the midpoint would be the mid-way between the farthest anterior and farthest posterior points of the plane and the mid-way between the lateral edges of the plane. Elliott [15] presented three examples for determining the reference in helmet design. The first method was based on the anatomical landmark of the glabella (in the front of the head between the brow ridges, directly above the nose) and the farthest distance from the glabella in the horizontal plane. The second method is based on the mid-point defined by the glabella and the nuchales. The third method used glabella, nuchales, and left and right tragions for determining the midpoint. It's more complex than the former two and adopted by the researcher.

In our view, alignment reference should be carefully chosen, and it should be based on the specific requirements of an application. Any man-made alignment reference should take the safety, comfort and other ergonomic factors into consideration; otherwise the data analysis results would be useless for the engineering product design. Specifically speaking, for head accommodation, the fitting of modern flight helmets not only means comfort, but also proper placement of accessorial components (e.g., earcups, helmet-mounted optics, etc.), stability, and proper location of center of gravity [17]. Such kind of helmets must provide protection of the whole head. In addition, they must satisfy evenly mass distribution in all directions of the head as much as possible, since stability is highly expected. Therefore, if the upper head samples were utilized in establishing sizing system for helmets emphasizing stability around the whole head, such as flight helmets, it's recommended the upper heads aligned at the centroid of the whole head. In contrast, if the upper head samples were used in helmet sizing system for infantryman, who emphasizes protection and fitting of the upper head, it's proper to align the head samples at the top of the head.

K-means clustering results of upper head samples, aligned at the head top and aligned at the centroid of the head have been demonstrated in this paper. The implication obtained from such a small CMAR value (only 53\%), as shown in Table 1, reveals that alignment method can lead to very different $3 \mathrm{D}$ shape clustering results. 


\section{Alignment method Aligned at head top}

Front view
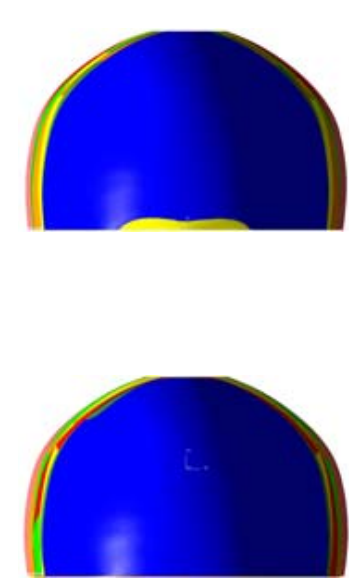

Side view
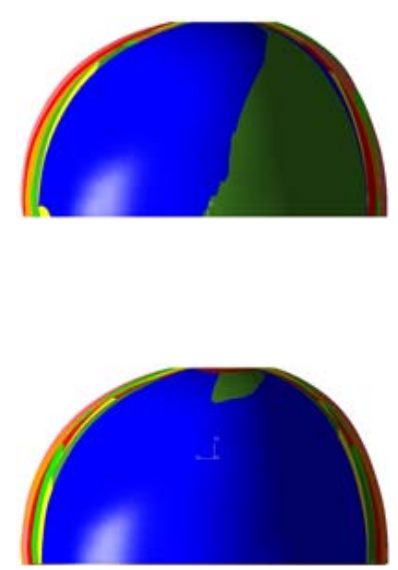

Bottom view

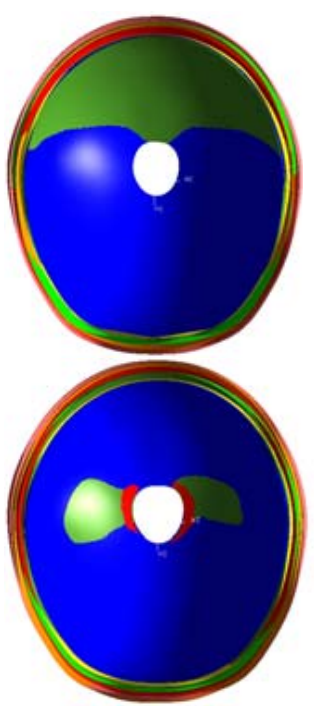

Fig. (2). Different views of the merged average upper heads of clusters.

Average sample of a cluster was used as the representative sample of the cluster. It was generated by averaging coordinates of corresponding points of all the samples belonging to the same cluster. Different views of the merged average upper head samples of the clusters are shown in Fig. (2). The difference among clusters can be seen from this figure. For example, from front and side views, the size and shape change from one cluster to another cluster can be easily found. The bottom view mainly reflects the bottom boundary difference.

\section{CONCLUSIONS}

Whereas plenty of effort has been devoted to anthropometric data analysis, few studies has focused on the selection of alignment reference. In our case study of 432 upper head samples with two different alignments, big difference (Cluster Membership Accuracy Rate only reaches to 53\%) has been observed between the two alignment methods. This implies that a suitable alignment reference should be selected when processing 3D anthropometric data for population grouping. The application background should be carefully considered when selecting alignment reference; otherwise, the results may lead to improper design on safety and/or fitting comfort.

\section{ACKNOWLEDGEMENT}

This work was supported by the National Natural Science Foundation of China (Grant No.70571045)

\section{REFERENCES}

Harrison CA, Robinette, KM. Principles of fit to optimize helmet sizing. Proceedings of the NATO Research Technology Organization's Human Factors \& Medicine Panel Symposium on Strategies to Maintain Combat Readiness during Extended Deployments-A Human Systems Approach; 2005.
[2] Witana CP, Xiong SP, Zhao JH, Goonetilleke RS. Foot measurements from three-dimensional scans: A comparison and evaluation of different methods. Int J Ind Ergon 2006; 36: 789-807.

[3] Au EYL, Goonetilleke RS. A qualitative study on the comfort and fit of ladies' dress shoes. Appl Ergon 2007; 38(6): 687-96.

[4] Lee HY, Hong KH. Optimal brassiere wire based on the 3D anthropometric measurements of under breast curve. Appl Ergon 2007; 38: 377-84.

[5] Zhuang Z, Bradtmiller B, Shaffer RE. New respirator fit test panels representing the current US civilian work force. J Occup Environ Hyg 2007; 4(9): 647-59.

[6] Chung MJ, Lin HF, Wang MJ. The development of sizing systems for Taiwanese elementary- and high-school students. Int J Ind Ergon 2007; 37: 707-16.

[7] Robinette KM, Blackwell S, Daanen H, et al. Technical report: AFRL-HE-WP-TR-2002-0169, CAESAR, Final Report, Volume I: Summary United States Air Force Research Lab, Human Effectiveness Directorate, Crew System Interface Division, Dayton Ohio 2002.

[8] Meunier P, Tack D, Ricci A, Bossi L, Angel H. Helmet accommodation analysis using 3D laser scanning, Appl Ergon 2000; 31: 3619.

[9] Hsiao HW, Whitestone J, Kau TY. Evaluation of Fall Arrest Harness Sizing Schemes. Hum Factors 2007; 49(3): 447-64.

[10] Rogers MS, Barr AB, Kasemsontitum B, Rempel DM. A threedimensional anthropometric solid model of the hand based on landmark measurements. Ergonomics 2008; 51; 4: 511-26.

[11] Richtsmeier JT, DeLeon VB, Lele S. The promise of geometric morphometrics. Yearb Phys Anthropol 2002; 45: 63-91.

[12] Cai XW, Li ZZ, Chang CC, Dempsey P. Analysis of alignment influence on $3 \mathrm{~d}$ anthropometric statistics. Tsinghua Sci Technol 2005; 10 (5): 623-6.

[13] Niu JW, Li ZZ, Salvendy G. Multi-resolution shape description and clustering of three-dimensional head data. Ergonomics, accepted.

[14] Chen X, Shi MW, Zhou H, Wang XT, Zhou GT. The standard head for sizing military helmets based on computerized tomography and the headform sizing algorithm. Acta Armamentarii 2002; 23(4): 476-80 (in Chinese).

[15] Elliott MG. Methodology for the sizing and design of protective helmets using three-dimensional anthropometric data. PH. D. dissertation, Colorado State University, Fort Collins, Colorado, USA 1998. 
[16] Huggins A. Derivation of helmet sizing criteria from a $3 \mathrm{~d}$ laser head scan database. Proceedings of the Design and Integration of Helmet Systems, Sheraton Tara Hotel, Framingham, MA, December 2-5: 1997.

[17] Robinette KM, Whitestone JJ. Methods for characterizing the human head for the design of helmets Technical report: AL-TR-1 992-0061 Wright-Patterson AFB OH: Armstrong Laboratory 1992.
[18]

Bradtmiller B, Beecher R. An approach to Creating ThreeDimensional Head Forms for Helmet Sizing and Design. Proceedings of the Thirty-First Annual Symposium, SAFE Association, Riviera Hotel, Las Vegas, Nevada, November 8-10: 1993; 244-9.

(c) Niu et al.; Licensee Bentham Open.

This is an open access article licensed under the terms of the Creative Commons Attribution Non-Commercial License (http://creativecommons.org/licenses/by$\mathrm{nc} / 3.0 /$ ) which permits unrestricted, non-commercial use, distribution and reproduction in any medium, provided the work is properly cited. 